\title{
Positron energy distribution in factorized trident process
}

\author{
A. I. Titov ${ }^{1}$, U. Hernandez Acosta ${ }^{2,3}$, B. Kämpfer ${ }^{2,4}$ \\ ${ }^{1}$ Bogoliubov Laboratory of Theoretical Physics, JINR, Dubna 141980, Russia \\ ${ }^{2}$ Helmholtz-Zentrum Dresden-Rossendorf, 01314 Dresden, Germany \\ ${ }^{3}$ Center for Advanced Systems Understanding, Helmholtz-Zentrum \\ Dresden-Rossendorf e.V. (HZDR) Untermarkt 20, 02826 Görlitz, Germany and \\ ${ }^{4}$ Institut für Theoretische Physik, TU Dresden, 01062 Dresden, Germany
}

(Dated: December 30, 2021)

\begin{abstract}
We estimate the energy distribution of positrons produced in the interaction of ultra-relativistic electrons with a high-intensity laser beam. The underlying trident process is factorized on the probabilistic level. That is, we deploy a two-step mechanism for the formation of electron-positron pairs. In the first step, a high-energy photon is produced as a result of nonlinear Compton scattering. In the second step, an electron-positron pair is created by the nonlinear (multi-photon) BreitWheeler process.
\end{abstract}

PACS numbers: 12.20.Ds, 13.40.-f, 23.20.Nx

Keywords: trident, nonlinear Compton scattering, nonlinear Breit-Wheeler pair production, strong-field QED

\section{INTRODUCTION}

Our study is devoted to an analysis of the energy spectra of positrons $\left(e^{+}\right)$produced in collisions of ultrarelativistic electrons $\left(e^{-}\right)$with a high-intensity laser beam $\left(\mathcal{L}\right.$, circular polarization), $e^{-}+\mathcal{L} \rightarrow e^{-^{\prime}}+e^{+} e^{-}$, where $e^{-\prime}$ is the recoil electron. This is the trident process which, analog to the nonlinear two-photon Compton process [1], is described within the Furry picture by a prototypical two-vertex diagram and its exchange part. Quantum interference effects make a throughout description quite challenging. Correspondingly, a series of papers, e.g. 4 14, analyzed in depth the many interesting facets of trident [15, 16] as a fundamental strongfield QED process. Trident is to be considered as the fist stage of the development of QED cascades and plays thus a prominent role, also for testing numerical simulation codes [17-19], in particular in the strong-field regime. The increasing interest is documented by a number of plans of experimental facilities 20 24 enabling high-precision measurements after the pioneering work of E-144 [25, 26] and successors [27, 28.

In view of these developments, an easily usable approximation of trident should be devised. Following suggestions in [7 9] and in line with earlier approaches 5. 6, 14, 25, we apply a folding model of type $\mathrm{n} \ell \mathrm{C} \otimes$ $\mathrm{n} \ell \mathrm{BW}$, i.e. gluing the probabilities of nonlinear Compton $(\mathrm{n} \ell \mathrm{C})$ and nonlinear Breit-Wheeler $(\mathrm{n} \ell \mathrm{BW})$ processes as depicted by the Furry picture diagrams

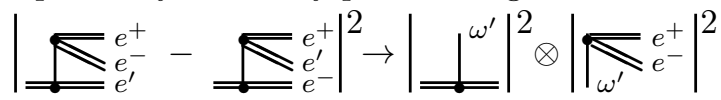

(dou-

ble lines: Volkov wave functions, vertical lines: photon propagator or real unpolarized photon with frequency $\left.\omega^{\prime}\right)$. This is the incoherent product of two one-vertex processes in the spirit of kinetic simulations, cf. [29, 30], which is realized here by the convolution

$$
\int d \omega^{\prime} \frac{d \Gamma_{n \ell C}^{e^{-}+\mathcal{L} \rightarrow \gamma^{\prime}\left(\omega^{\prime}\right)+e^{-\prime}}}{d \omega^{\prime}} \Gamma_{n \ell B W}^{\gamma^{\prime}\left(\omega^{\prime}\right)+\mathcal{L} \rightarrow e^{+} e^{-}}
$$

of $\mathrm{n} \ell \mathrm{C}$ and $\mathrm{n} \ell \mathrm{BW}$ probabilities or rates $\Gamma_{n \ell C, n \ell B W}$ (see also equation (21) in [5] and equation (30) in [6]). The integration over the energy $\omega^{\prime}$ of the intermediate photon reduces strongly the impact of $\omega^{\prime}$-local structures of the individual rates. The recent references [31, 32 discuss various approximation schemes of one-vertex processes and their capabilities to catch the rich structures in laser pulses. The convolution (1) make them less severe.

Our focus is on the positron energy distribution. The pair production probabilities have been considered in 33. within the same folding model. Positrons are best identifiable and do not suffer from distinguishing the recoil electron from the pair electron. The selected kinematics is motivated by plans of the LUXE collaboration [22, 23 . The considered laser intensities are high but not ultrahigh, so that we are working in a regime where the RitusNaroshny conjecture does not apply, therefore enabling the safe use of the Furry picture. The two building blocks of our approach, i.e. $\mathrm{n} \ell \mathrm{C}$ and $\mathrm{n} \ell \mathrm{BW}$, have been elaborated long ago by Nikishov and Ritus, see 34 .

Our short note is organized as follows. In section II we recall the main formulas for nonlinear Compton and Breit-Wheeler processes w.r.t. to the easy application in calculating the energy distribution of the produced positrons in electron-laser collisions which we consider in section III Besides that also the laser intensity dependence is discussed and a brief remark on the "boiling point of the QED vacuum" is added there. Our summary and remarks for further developments are given in section [V] Band width effects are considered in appendix A for linear trident. 


\section{NONLINEAR ONE-VERTEX PROCESSES}

Analog to kinetic theory on probabilistic level as causal reaction network we approximate the factorized trident process by multiple emission as incoherent combination of one-vertex processes. Albeit nonlinear Compton and nonlinear Breit-Wheeler processes are related by crossing symmetry on amplitude level, their final phase spaces are different and the physically allowed regions in the Mandelstam plane have different shapes, as exhibited in figure 1 in [35. Our ansatz in section III below, based on the convolution (1), needs the rates $\frac{d \Gamma_{n \ell C}\left(\omega^{\prime} \mid E_{e}\right)}{d \omega^{\prime}}$ and $\frac{d \Gamma_{n \ell B W}\left(E^{+} \mid \omega^{\prime}\right)}{d E^{+}}$which depend parametrically on laser frequency and intensity. The lab. frame kinematic quantities $E_{e}$ (in-electron energy), $\omega^{\prime}$ (hard-photon energy) and $E^{+}$(out-positron energy) uncover certain ranges: $0<\omega^{\prime}<E_{e}(\mathrm{n} \ell \mathrm{C})$ and $0<E^{+}<\omega^{\prime}(\mathrm{n} \ell \mathrm{BW})$, suggesting representations of out-states quantities scaled by in-state quantities, i.e. $0<\omega^{\prime} / E_{e}<1$ and $0<E^{+} / \omega^{\prime}<1$ and finally $0<E^{+} / E_{e}<1$. The different dependencies of $\mathrm{n} \ell \mathrm{C}$ and $\mathrm{n} \ell \mathrm{BW}$ rates on (out $\mid$ in) quantities also enable different approximations which we recollect in the subsequent subsections on the basis of adapted textbook formulas.

For the classical laser intensity parameter we use the Lorentz and gauge invariant variable $\xi=|e| \mathcal{E} /(m \omega)$, expressed by quantities in the lab. system: $\mathcal{E}$ - electric laser field strength (peak value), $\omega$ - the laser's central frequency; $-|e|$ and $m$ stand for the electron charge and mass, respectively, and $\alpha=e^{2} / 4 \pi \approx 1 / 137$ is the finestructure constant. We use natural units with $\hbar=c=1$.

\section{A. Nonlinear Compton scattering}

The following notation is employed for the nonlinear Compton process $e+\mathcal{L} \rightarrow e^{\prime}+\gamma^{\prime}: p$ is the $i n$-electron fourmomentum, $k$ the laser photon four-momentum, and $k^{\prime}$ the out-photon's $\left(\gamma^{\prime}\right)$ four-momentum. Quantities in the lab. frame $E_{e}, \omega$ and $\omega^{\prime}$ denoting the incoming (beam) electron energy mentioned above, frequency of the above mentioned laser beam and Compton-produced photons, respectively. For simplicity, we restrict ourselves on headon electron-laser collisions and leave the explication of formulas for the experimentally relevant case of a finite inclination of both beams to later work. The laser frequency is chosen as $\omega=1.55 \mathrm{eV}$.

The one-photon emission rate in a monochromatic, circularly polarized background (laser) field is $33,34,36$.

$$
\begin{gathered}
\frac{d \Gamma_{n \ell C}}{d \omega^{\prime}}=\frac{\alpha m^{2}}{E_{e}^{2}} \sum_{n=n_{\text {min }}}^{\infty} \mathcal{F}_{n}(z, u, \xi) \\
\mathcal{F}_{n}=-J_{n}^{2}(z)+\xi^{2} w(u)\left(\left[\frac{n^{2}}{z^{2}}-1\right] J_{n}^{2}(z)+{J_{n}^{\prime}}^{2}(z)\right)(3)
\end{gathered}
$$

where $w(u)=1+\frac{u^{2}}{2(1+u)}$ and

$$
\begin{aligned}
& n_{\text {min }}=\left\lceil 1+\frac{m^{2}\left(1+\xi^{2}\right) \omega^{\prime}}{4 \omega E_{e}\left(E_{e}-\omega^{\prime}\right)}\right\rceil \\
& z=\frac{\xi^{2} \sqrt{1+\xi^{2}}}{\chi} \sqrt{u\left(u_{n}-u\right)}, \quad \chi=\xi k \cdot p / m^{2}, \\
& u=k \cdot k^{\prime} / k \cdot p^{\prime} \approx \omega^{\prime} /\left(E_{e}-\omega^{\prime}\right), u_{n}=\frac{2 n \chi}{\xi\left(1+\xi^{2}\right)}
\end{aligned}
$$

$J_{n}$ and $J_{n}^{\prime}$ denote Bessel function of first kind and its derivative, respectively, and $\lceil\cdot\rceil$ stands for the ceiling function. Some approximation of (3) is provided by utilizing an asymptotic expression of the Bessel function

$$
J_{n}(z) \approx(2 \pi n \tanh a)^{-1} \mathrm{e}^{2 n(a-\tanh a)}
$$

with $\tanh a=\sqrt{1-z^{2} / n^{2}}$ valid at $\xi \gg 1$. In numerically evaluating the differential rate of $\mathrm{n} \ell \mathrm{C}$, the "large- $\xi$ approximation" [34] is often convenient:

$$
\frac{d \Gamma_{n \ell C}}{d \omega^{\prime}} \approx-\frac{\alpha m^{2}}{\pi E_{e}^{2}}\left\{\int_{z}^{\infty} d y \Phi(y)+\frac{2}{z} w(u) \Phi^{\prime}(z)\right\},
$$

where $\Phi(z)$ and $\Phi(z)^{\prime}$ stand for the Airy function and its derivative with arguments $z=(u / \chi)^{2 / 3}$.

The differential distributions $d \Gamma_{n \ell C} / d \omega^{\prime}$ for various initial electron energies $E_{e}$ and laser intensities $\xi$ are exhibited in Fig. 1.

The solid curves depict the monochromatic model, Eqs. (2, 3), and the dotted and dashed curves correspond to the approximations (7) and (8), respectively. Results depicted by solid and dotted curves are close to each other in the considered region of $E_{e}, \xi$ and $\omega^{\prime}$ At $\xi \geq 3$ and $\omega^{\prime} \geq 0.1 E_{e}$, the solid and dashed curves are indistinguishable. One can see a monotonic decrease of the rate with increasing $\omega^{\prime}$ and some plateau at $\omega^{\prime} \sim E_{e} / 2$. The width of the plateau increases with increasing $E_{e}$. The harmonic structures of Eqs. (2, 3) are visible at low values of $\omega^{\prime}$ and extend only for small values of $\xi$ towards moderate values of $\omega^{\prime}$. Despite the assumptions made in deriving the approximation Eq. (8) 34 the agreement with Eq. (3) is impressive for hard photons at $\xi>1$.

When considering a laser pulse, instead of the monochromatic background field, an additional scale enters, which characterizes, e.g. the pulse duration. Due to interference effects, the out-photon spectra may acquire more complex shapes with nonlinear dependencies on pulse shape and duration. There is extended literature on this and attempts of useful approximation schemes. We emphasize again that, in our positron number estimate below, the rate $\frac{d \Gamma_{n \ell C}\left(\omega^{\prime} \mid E_{e}\right)}{d \omega^{\prime}}$ is to be integrated over $\omega^{\prime}$ with the weight $\frac{d \Gamma_{n \ell B W}\left(E^{+} \mid \omega^{\prime}\right)}{d E^{+}}$at given $E^{+}$. In so far, the (fine or harmonic) structures in $\frac{d \Gamma_{n \ell C}\left(\omega^{\prime} \mid E_{e}\right)}{d \omega^{\prime}}$ do not matter. The agreement of the differential cross section for a pulse (see figure 8 in [36]) with the monochromatic spectrum according to Eqs. (2) 3) support that claim. As a consequence, Eqs. (2, 3, 7, 8) provide useful ingredients of our $\mathrm{n} \ell \mathrm{C} \otimes \mathrm{n} \ell \mathrm{BW}$ folding model. 

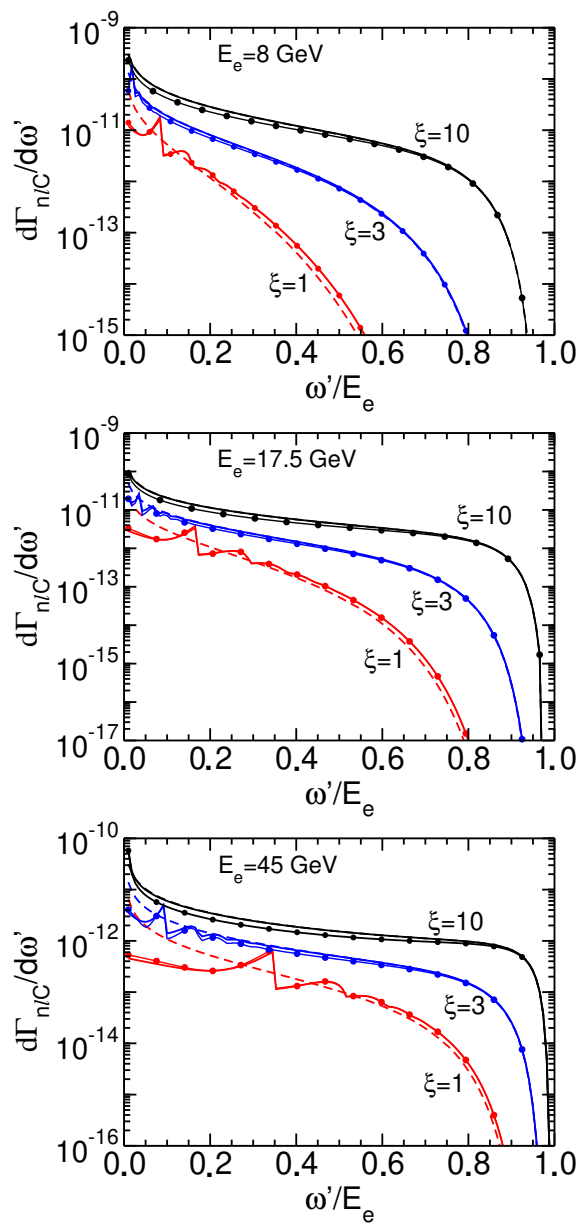

FIG. 1: (Color online) The dimensionless differential rate $d \Gamma_{n \ell C} / d \omega^{\prime}$ as a function of $\omega^{\prime}$ (scaled by $E_{e}$ ) for electron beam energies $E_{e}=8 \mathrm{GeV}$ (top panel), $17.5 \mathrm{GeV}$ (middle panel) and $45 \mathrm{GeV}$ (bottom panel) at different values of $\xi=1$ (red), 3 (blue) and 10 (black). The solid curves depict the monochromatic model Eqs. (2, 3), while dotted curves use the approximation Eq. (7). Dashed curves are for the approximation Eq. (8). The solid and dotted curves display harmonic structures at low values of $\omega^{\prime}$ and $\xi$.

\section{B. Nonlinear Breit-Wheeler process}

The differential rate of produced positrons in nonlinear (essentially multi-photon) Breit-Wheeler process $\gamma^{\prime}+\mathcal{L} \rightarrow e^{+} e^{-}$for given four-momentum $k^{\prime}$ (in-photon $\gamma^{\prime}$, frequency $\omega^{\prime}$ ) and $k$ (laser, frequency $\omega$ ) reads for head-on collisions 34

$$
\begin{gathered}
\frac{d \Gamma_{n \ell B W}}{d E^{+}}=\frac{\alpha m^{2}}{\omega^{\prime 2}} \sum_{n=n_{\min }}^{\infty} \mathcal{J}_{n}(z, u, \xi), \\
\mathcal{J}_{n}=J_{n}^{2}(z)+\xi^{2} w(u)\left(\left[\frac{n^{2}}{z^{2}}-1\right] J_{n}^{2}(z)+J_{n}^{\prime 2}(z)\right)(10)
\end{gathered}
$$

where the definitions 12,13 apply for $z$ and $u_{n}$ entering $\mathcal{J}_{n} 10$ with $\omega_{ \pm}^{\prime}=\omega^{\prime} \pm n \omega, q=\sqrt{q_{0}^{2}-m^{2}\left(1+\xi^{2}\right)}$, where $E^{+}$is the positron energy, $w(u)=(2 u-1)$ and

$$
\begin{aligned}
n_{\min } & =\left[1+\frac{m^{2}\left(1+\xi^{2}\right) \omega^{\prime}}{4 \omega E^{+}\left(\omega^{\prime}-E^{+}\right)}\right\rceil \\
z & =\frac{2 \xi n}{\sqrt{1+\xi^{2}}} \sqrt{\frac{u}{u_{n}}\left(1-\frac{u}{u_{n}}\right)} \\
u & =\frac{\omega^{\prime 2}}{4 E^{+}\left(\omega^{\prime}-E^{+}\right)}, \quad u_{n}=\frac{n \omega \omega^{\prime}}{m^{2}\left(1+\xi^{2}\right)}
\end{aligned}
$$

Some approximation is based on Eq. (10) with (7) yielding

$$
\mathcal{J}_{n}(z, u, \xi) \approx \frac{1}{2 \pi} \mathrm{e}^{2 n(a-\tanh a)} \frac{1+2 \xi^{2}(2 u-1) \sinh ^{2} a}{n \tanh a} .
$$

Analog to Eq. (8) one can utilize the approximation 34

$$
\frac{d \Gamma_{n \ell B W}}{d E^{+}} \approx \frac{\alpha m^{2}}{\pi \omega^{\prime 2}}\left\{\int_{z}^{\infty} d y \Phi(y)-\frac{2}{z}(2 u-1) \Phi^{\prime}(z)\right\}
$$

with $z=\left(4 u / \chi_{\gamma}\right)^{2 / 3}$ and $\chi_{\gamma}=\xi k \cdot k^{\prime} / m^{2}$, which both have been employed, e.g. in [5, 6].

The dependence of the rate $d \Gamma_{n \ell B W} / d E^{+}$as a function of $\omega^{\prime}$ for fixed positron energy $E^{+}=E_{e} / 2$ is exhibited in Fig. 2. Since $E^{+}<\omega^{\prime}$, only the range $0.5<\omega^{\prime} / E_{e}$ is accessible. The small (large)- $\omega^{\prime}$ range becomes accessible for small (large) values of $E^{+}$. Note the opposite tendencies of the Compton and Breit-Wheeler rates as a function of $\omega^{\prime}$, making the details of small- $\omega^{\prime}$ and large- $\omega^{\prime}$ distributions irrelevant when considering their product in convoluting them. The laser intensity dependency is also very strong in the considered parameter range.

Some information on the $E^{+}$dependence of $d \Gamma_{n \ell B W} / d E^{+}$at fixed values of $\omega^{\prime}$ is given in the left column of Fig. 3 for $E_{e}=17.5 \mathrm{GeV}$ at different values $\xi$. The symmetric energy distributions have a pronounced maximum at $E^{+}=\omega^{\prime} / 2$ and monotonically decrease for $E^{+} \rightarrow 0$ or $E^{+} \rightarrow \omega^{\prime}$, thus evidencing the relevant positron energy range. To quantify the positron polar-angular distribution and to characterize the relevant range we exhibit these in the right column of Fig. 3. The angular distributions exhibit a dead cone at small angles $\Theta^{\prime}$ relative to the $\omega^{\prime}$ photon direction, i.e. at $\left(1-\cos \Theta^{\prime}\right) \rightarrow 0$, similar to the nonlinear Compton process [35]. The dead cone effect becomes sharper with increasing value of $\xi$. The displayed angular-differential rate is determined by

$$
\frac{d \Gamma_{n \ell B W}}{d \cos \Theta^{+}}=\frac{\alpha m^{2}}{\omega^{\prime}} \sum_{n=n_{\min }}^{\infty} \frac{q^{2}}{\left|q \omega_{+}^{\prime}-q_{0} \omega_{-}^{\prime} \cos \Theta^{+}\right|} \mathcal{J}_{n}
$$



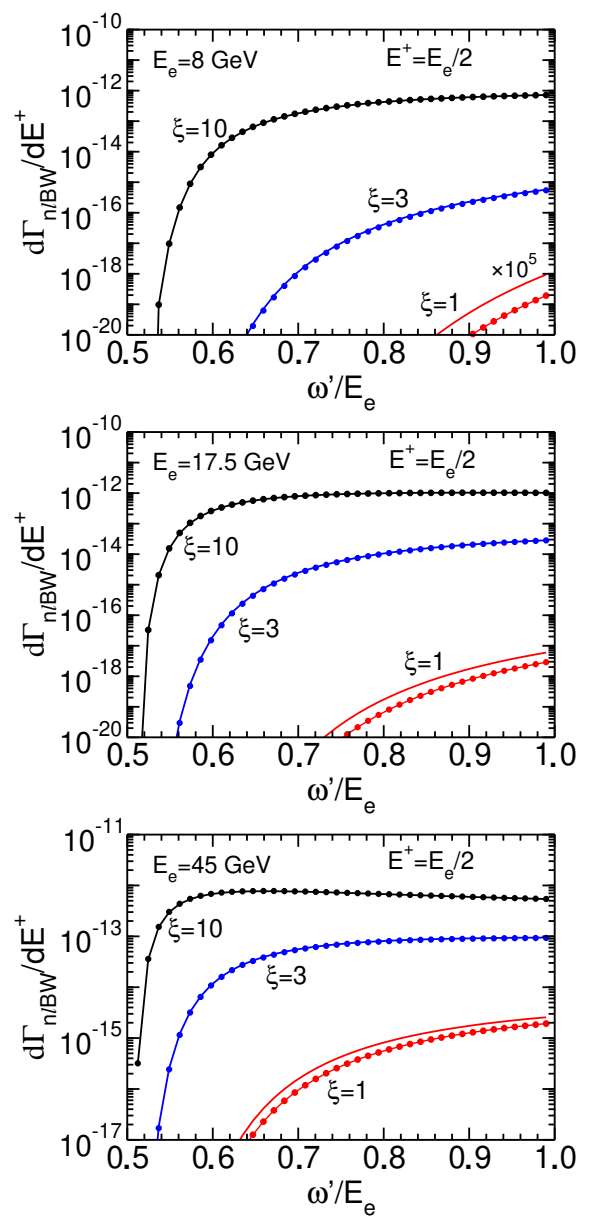

FIG. 2: (Color online) The dimensionless differential rate $d \Gamma_{n \ell B W} / d E^{+}$as a function of $\omega^{\prime}$ (scaled by $E_{e}$ ) for positron energies $E^{+}=E_{e} / 2$ for $E_{e}=8 \mathrm{GeV}$ (top panel), $17.5 \mathrm{GeV}$ (middle panel) and $45 \mathrm{GeV}$ (bottom panel) at different values of $\xi=1$ (red), 3 (blue) and 10 (black). Results using either Eqs. 9 10 (solid curves) or 9, 14 (dashed curves) are not distinguishable on the given scale. The approximation 15 is displayed by dotted curves.

$q_{0}=A+\sqrt{A^{2}-B}$, as well as

$$
\begin{aligned}
n_{\text {min }} & =\left[1+\frac{m^{2}\left(1+\xi^{2}\right)}{\omega \omega^{\prime}}\right\rceil, \\
u & =\frac{\omega^{\prime 2}}{\left(q_{0}+q \cos \Theta^{+}\right)\left(2 \omega^{\prime}-q_{0}-q \cos \Theta^{+}\right)}, \\
A & =\frac{2 n \omega \omega^{\prime} \omega_{+}^{\prime}}{\omega_{+}^{\prime 2}-\omega_{-}^{\prime 2} \cos ^{2} \Theta^{+}}, \\
B & =\frac{m^{2}\left(1+\xi^{2}\right) \omega_{-}^{\prime 2} \cos ^{2} \Theta^{+}+4\left(n \omega \omega^{\prime}\right)^{2}}{\omega_{+}^{\prime 2}-\omega_{-}^{\prime 2} \cos ^{2} \Theta^{+}} .
\end{aligned}
$$

\section{ESTIMATE OF POSITRON PRODUCTION}

The above recollected rates for monochromatic background (laser) fields may be realized by a very long
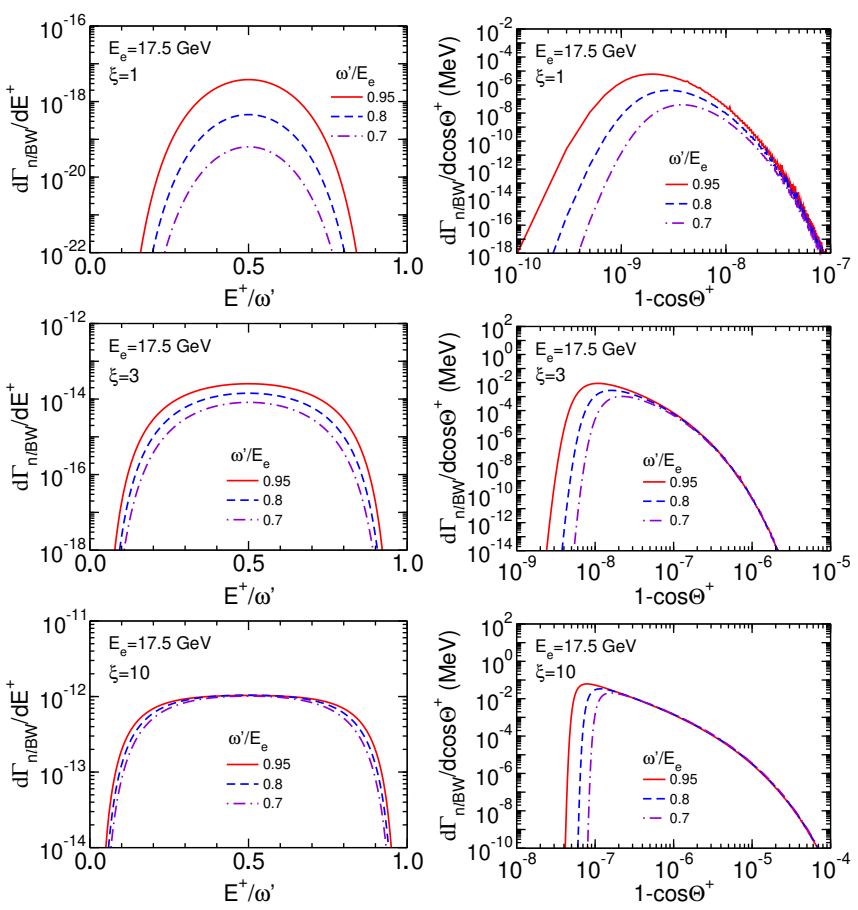

FIG. 3: (Color online) Positron production rates $d \Gamma_{n \ell B W} / d E^{+}$as a function of positron energy $E^{+}$(scaled by $\omega^{\prime}$ ) (left row) and $d \Gamma_{n \ell B W} / d \cos \Theta^{+}$as a function of the positron lab. angle $\Theta^{+}$relative to the $\omega^{\prime}$-photon direction (right row) at laser intensity parameters $\xi=1$ (top panel), 3 (middle panel) and 10 (bottom panel) and various energies of the probe photon $\omega^{\prime}$ in units of $E_{e}=17.5 \mathrm{GeV}$. In calculation we use Eq. (14.

flattop section of the envelope. To be specific consider a plane-wave laser model with electromagnetic vector potential $A(\phi) \propto f(\phi) \cos (\phi)$ with invariant phase $\phi=\omega(t+z)$, i.e. a beam moving along $z$ axis to left, and lines of constant phase are inclined by $45^{\circ}$ in the observer's space-time $(z-t)$ diagram. An idealized flattop envelope would be $f(\phi)=\sqcap(\phi, \Delta \phi)$ with $\sqcap=1$ for $\phi \in[0, \Delta \phi]$ and zero elsewhere, i.e. the number of oscillations within the pulse is $N=\Delta \phi / 2 \pi$. (Such an envelope has been considered in 32 and 37] for $\mathrm{n} \ell \mathrm{C}$ and $\mathrm{n} \ell \mathrm{BW}$, respectively, with emphasis on the soft part of the distributions and comparison of various approximation schemes.) Despite intricate effects of finite-time laser pulses [32], a simplified estimate of the number of produced quanta would be to use the monochromatic rate multiplied by the pulse duration. In such a spirit we estimate the number of produced positrons by convoluting the rate of produced hard photons, emitted by a highenergy electron - traversing a pulse on the trajectory $t \approx z$ near light cone thus crossing the invariant phase lines $\phi=0 \cdots \Delta \phi-$ with the rate of positrons produced by these photons later on within the same pulse. Let be the relevant time intervals $T_{C, B W}$ for the respective $\mathrm{n} \ell \mathrm{C}$ and $\mathrm{n} \ell \mathrm{BW}$ processes. Then the energy distribution of positrons in the folding model $\mathrm{n} \ell \mathrm{C} \otimes \mathrm{n} \ell \mathrm{BW}$ may be 
expressed as

$$
\frac{d N^{e^{+}}}{d E^{+}}=\int_{0}^{E_{e}} d \omega^{\prime} \int_{0}^{T_{C}} d t \frac{d \Gamma_{n \ell C}\left(\omega^{\prime}, t\right)}{d \omega^{\prime}} \int_{t}^{T_{B} W} d t^{\prime} \frac{d \Gamma_{n \ell B W}\left(\omega^{\prime}, t^{\prime}\right)}{d E^{+}},
$$

where $d \Gamma_{n \ell C}\left(\omega^{\prime}, t\right) / d \omega^{\prime}$ is the rate of photons per frequency interval $d \omega^{\prime}$ emerging from one electron undergoing Compton scattering at time $t \in\left[0, T_{C}\right]$, and $d \Gamma_{n \ell B W}\left(\omega^{\prime}, t^{\prime}\right) / d E^{+}$is the the differential rate of BreitWheeler pairs generated by a probe photon of frequency $\omega^{\prime}$ at lab. frame time $t^{\prime} \in\left[t, T_{B W}\right]$. Formally, $\Gamma_{n \ell C}\left(\omega^{\prime}, t\right) \rightarrow \Gamma_{n \ell C}\left(\omega^{\prime}\right) \sqcap\left(t, T_{C}\right)$ and $\Gamma_{n \ell B W}\left(\omega^{\prime}, t^{\prime}\right) \rightarrow$ $\Gamma_{n \ell B W}\left(\omega^{\prime}\right) \sqcap\left(t^{\prime}, T_{B W}\right)$. The above picture of an electron traversing a laser pulse in head-on geometry near to light cone means $T_{C}=T_{B W} \equiv T_{0}=\pi N / \omega$. (The pulse duration for an observer at rest in the $z$-t Minkowski frame is $2 T_{0}$. On the trajectory $z \approx t$, the invariant phase is $\phi \approx 2 \omega t$, therefore, $\sqcap\left(t, T_{0}\right) \rightarrow \sqcap(\phi, \Delta \phi)$.) Neglecting spatio-temporal variations within the pulse by using Eqs. (2) and (9), our final formula becomes

$$
\frac{d N^{e^{+}}}{d E^{+}}=F_{t} \int_{E^{+}}^{E_{e}} d \omega^{\prime} \frac{d \Gamma_{n \ell C}\left(\omega^{\prime}\right)}{d \omega^{\prime}} \frac{d \Gamma_{n \ell B W}\left(\omega^{\prime}, E^{+}\right)}{d E^{+}}
$$

with $F_{t}=N_{0}^{e} T_{C}\left(T_{B W}-T_{C} / 2\right)=T_{0}^{2} / 2$, i.e. a quadratic dependence on the pulse length via $N^{2}$. Such an estimate neglects the attenuation of the primary electron beam towards emitting a hard photon at $t \approx z$ (which can be cured by a Glauber eikonal factor), a formation time for separating the recoil electron and the on-shell photon (which is here assumed to continue on the straight trajectory), and the poor approximation of the photon number distribution in a finite pulse by neglecting bandwidth effects (see Appendix A for genuine bandwidth effects in the weak-field trident process). Obviously, the separation in one-step and the here only partially uncovered two-step processes on probabilistic level is rather crude. Therefore, in our estimates we either use $T_{0}=2 \pi / \omega$ (see Appendix B for a comparison with E-144 data) and attach to the Compton rate the number $N_{0}^{e}=6 \times 10^{9}$ of primary electrons per bunch or scale out the normalization factor $F_{t}$ 38.

Our estimate of the positron distribution $d N^{e^{+}} / d E^{+}$ is exhibited in Fig. 4. For $\mathrm{n} \ell \mathrm{Co}$ and $\mathrm{n} \ell \mathrm{BW}$ vertices we us Eqs. (8) and (9.14), respectively. The positron spectra display asymmetric bump-like distributions with the maximum shifted to smaller energies, i.e. to $E^{+}<E_{e} / 2$. At large beam-electron energy the bump like distributions become flatter and slightly inclined. The available phase space, $0<E^{+}<E_{e}$, becomes more and more uncovered with increasing values of $\xi$. Note the enormous sensitivity against variations of the laser intensity $\xi$.

To quantify the $\xi$ dependence we display in Fig. 5 the scaled positron number for three electron beam energies. To convert to absolute numbers one has to multiply by the beam electron number per bunch and adjust $T_{C}$
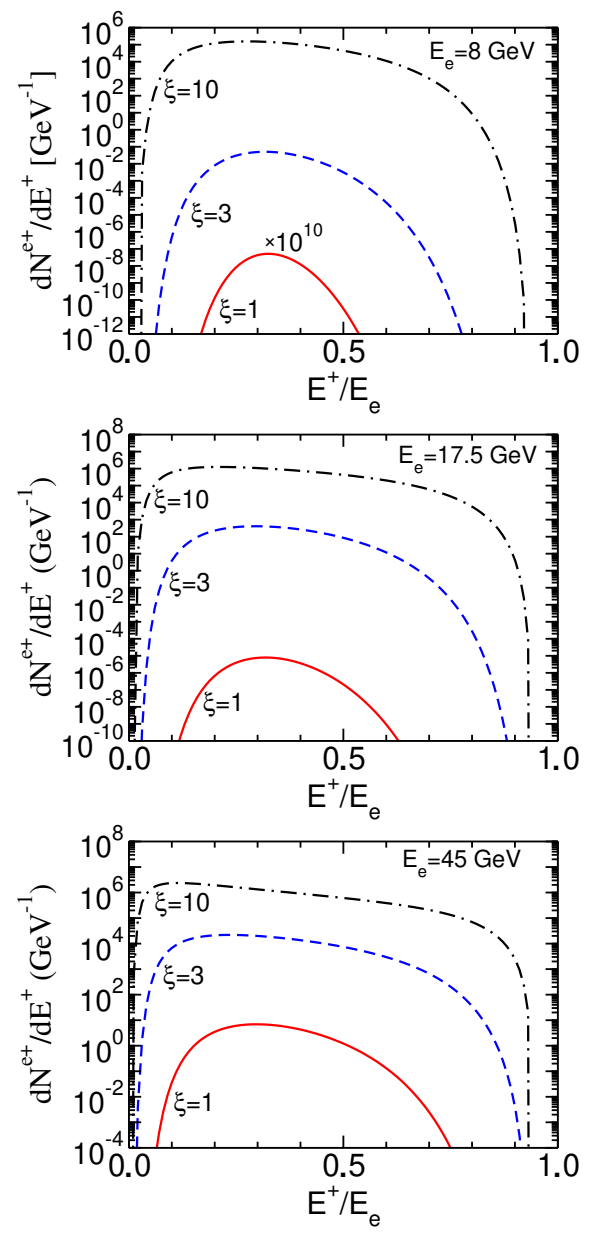

FIG. 4: (Color online) The distribution of positrons $d N^{e^{+}} / d E^{+}$as a function of lab. energy $E^{+}$at different values of $\xi=1$ (red), 3 (blue dashed) and 10 (black dot-dashed) for electron beam energies $E_{e}=8 \mathrm{GeV}$ (top panel), $17.5 \mathrm{GeV}$ (middle panel) and $45 \mathrm{GeV}$ (bottom panel) with $N_{0}=6 \times 10^{9}$. The harmonic structures of $d \Gamma_{n \ell C} / d \omega^{\prime}$ seen in Fig. 1 at small values of $\omega^{\prime}$ are irrelevant for $d N^{e^{+}} / d E^{+}$since $d \Gamma_{n \ell B W} / d \omega^{\prime}$ (see Fig. 2) is exceedingly small there.

and $T_{B W}$ in Eq. 21) which determine the factor $F_{t}$ in Eq. (22). The top panel is for the laser intensity range to be uncovered by LUXE - E-320. Using the above formalism also for ultra-high intensities, the scaled positron number converge at $\xi>50$ to a unique value irrespective of the electron beam energy $E_{e}$. Such a behavior is consistent with the stabilization phenomenon discussed in [39].

The laser intensity parameter may be noted as $\xi=$ $\frac{m}{\omega} \frac{\mathcal{E}}{\mathcal{E}_{\text {crit }}}$ with the (critical) Sauter-Schwinger electric field strength $\mathcal{E}_{\text {crit }}=m^{2} /|e| \approx 1.3 \times 10^{18} \mathrm{~V} / \mathrm{m}$. According to [40, "Measuring the Boiling Point of the Vacuum of Quantum Electrodynamics", one can perform a deconvolution of data to get an experimental access to $\mathcal{E}_{\text {crit }}$, supposed all other parameter are under control. Given the strong $\xi$ dependence of the individual contributions in $\mathrm{n} \ell \mathrm{C}(\xi) \otimes \mathrm{n} \ell \mathrm{BW}(\xi)$, see Figs. 1 and 2, one can anal- 

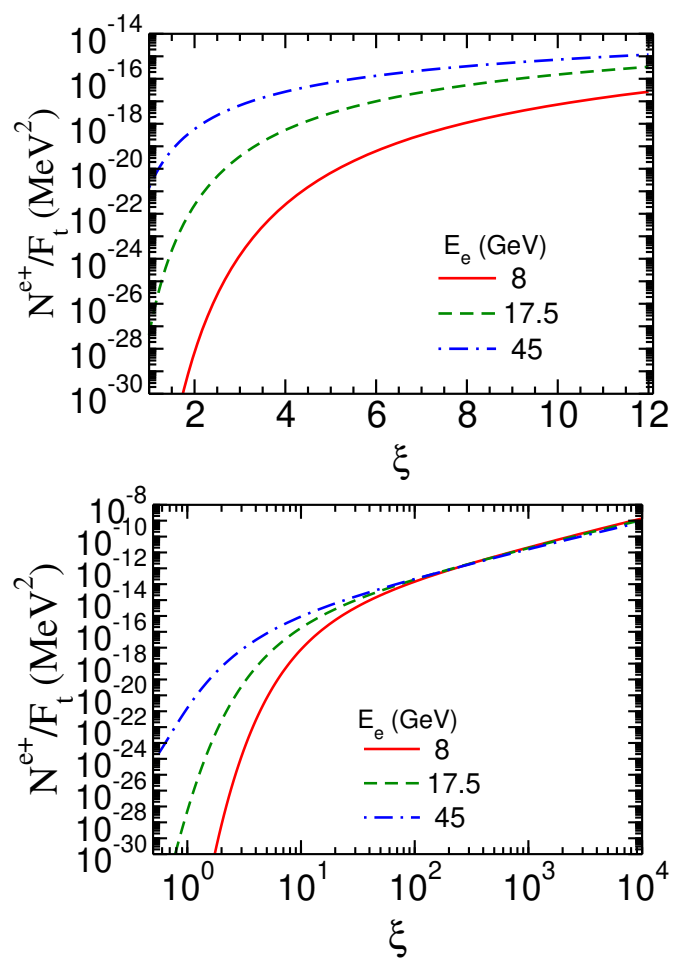

FIG. 5: (Color online) The scaled number of positrons $N^{e^{+}} / F_{t}$ as a function of laser intensity parameter $\xi$ for electron beam energies $E_{e}=8 \mathrm{GeV}$ (solid red), $17.5 \mathrm{GeV}$ (dashed green) and $45 \mathrm{GeV}$ (dot-dashed blue). Top panel: for intensity parameter $\xi$ in the LUXE - FACET-II (E-320) range. Bottom panel: for a large range of $\xi$. Note the value of the above given factor $F_{t} \omega^{2} \approx 20 N_{0}$.

ogously test the dependence of the complete two-step positron number $N^{e^{+}}$on $\mathcal{E}_{\text {crit }}$. A useful quantity is the ratio $N^{e^{+}}\left(\epsilon \mathcal{E}_{\text {crit }}\right) / N^{e^{+}}\left(\mathcal{E}_{\text {crit }}\right)=N^{e^{+}}(\xi / \epsilon) / N^{e^{+}}(\xi)$ as a function of $\xi$ with all other kinematic quantities fixed, see Fig. 6. Due to the exponential suppression of both the nonlinear Compton process [35] and the nonlinear Breit-Wheeler process [34, 41], which is encoded in the strong $\xi$ dependence, such a ratio exhibits in fact a stark sensitivity on variations of $E_{\text {crit }}$ on the $10 \%$ level.

\section{SUMMARY}

In summary we calculate, within a folding model $\mathrm{n} \ell \mathrm{C}$ $\otimes \mathrm{n} \ell \mathrm{BW}$ as approximation of the trident process, the energy distributions of positrons produced in collisions of ultra-relativistic electrons with a high-intensity laser beam for different electron beam energies and intensities of the laser beam. As a prerequisite we evaluate the gross shape (by ignoring the laser pule shape and thus not catching the complex interference pattern) of photon energy distributions from $\mathrm{n} \ell \mathrm{C}$, which are also experimentally accessible [42]. Our predictions are for parameters motivated by forthcoming experiments with $\xi=1 \cdots 10$,

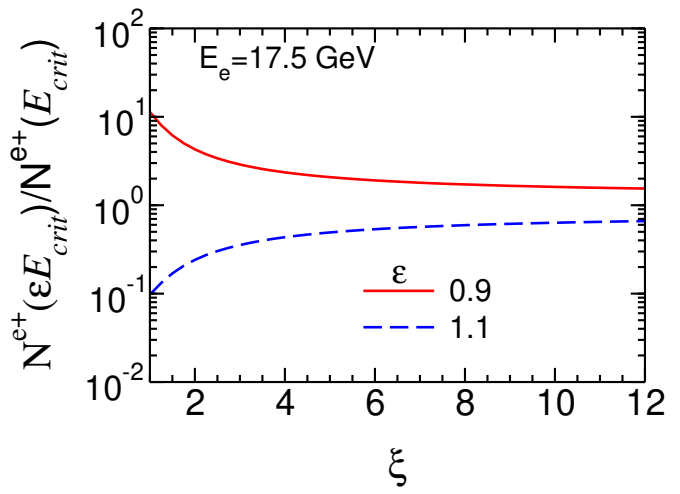

FIG. 6: (Color online) The ratio $N^{e^{+}}\left(\epsilon \mathcal{E}_{\text {crit }}\right) / N^{e^{+}}\left(\mathcal{E}_{\text {crit }}\right)$ as a function of $\xi$ for $E_{e}=17.5 \mathrm{GeV}$. Upper (lower) curve: $\epsilon=0.9$ (1.1). Both curves approach unity for large values of $\xi$.

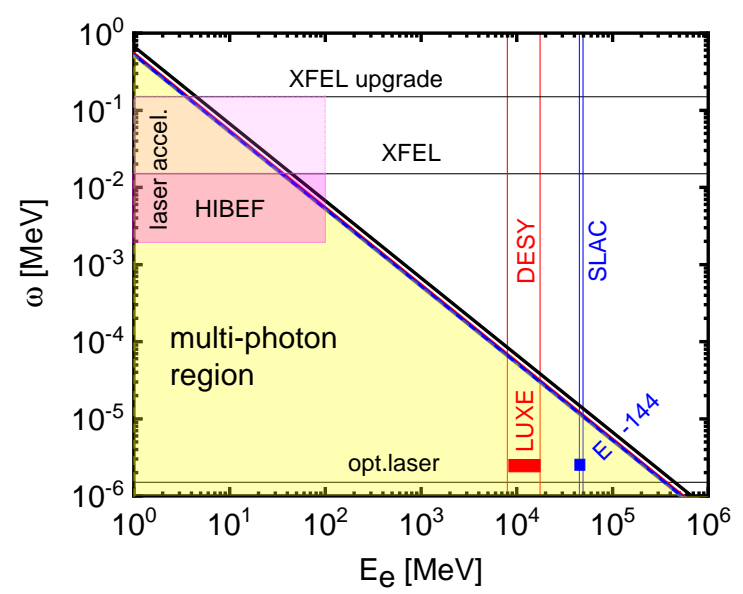

FIG. 7: (Color online) Trident landscape over the $\omega-E_{e}$ plane. The near-by diagonal lines depict the loci of $\sqrt{s} / m=3.353$, $3.05,3$ and 2.95 (from top to bottom) linear trident processes. The yellow region is below the threshold, i.e. accessible only by multi-photon effects in the nonlinear trident or by bandwidth effects in linear trident. Thin horizontal lines mark $1.5 \mathrm{eV}, 15 \mathrm{keV}$ and $150 \mathrm{keV}$ as representatives of optical lasers, XFEL and possible XFEL upgrade. Thin vertical lines bracket the energies of the Europaen XFEL electron driver beam (DESY) and Stanford linear accelerator (SLAC). Boxes filled with color indicate the previous E-144 (blue) and future LUXE (red) experiments as well as possible options at HIBEF (magenta) when using laser accelerated electrons with $E_{e}<100 \mathrm{MeV}$.

in particular at LUXE and FACET-II (E-320). While the employed folding model is related to the two-step part of trident in product approximation, the interesting one-step contribution should manifest itself as deviation. Our model may serve as reference easy to handle in such a search for genuine strong-field QED effects.

In future developments one may envisage triple-vertex processes, e.g. irradiating laser-accelerated electrons in the laser focus by a high-energy XFEL beam, XFEL + 
$\left(e^{-}+\mathcal{L}\right) \rightarrow e^{-^{\prime}}+e^{+} e^{-}$. This XFEL-induced trident is a nonlinear virtual Compton process, which is accessible to the HIBEF collaboration [43, in particular with an upgrade of the European XFEL. (The corresponding theory of the nonlinear real Compton sub-process, XFEL $+\left(e^{-}+\mathcal{L}\right) \rightarrow e^{-^{\prime}}+\gamma^{\prime}$, has been already dealt with in collinear kinematics [44.) An intermediate configuration could investigate the process XFEL $+e_{\mathcal{L}}^{-} \rightarrow$ $e^{-^{\prime}}+e^{+} e^{-}$, again with laser-accelerated electrons $\left(e_{\mathcal{L}}^{-}\right)$, but outside the laser focus. Since in the present setting the XFEL intensity parameter $\xi$ is small, $\xi_{\text {XFEL }} \ll 1$, the multi-photon effects - which are decisive in section III - are negligible. The threshold electron energy is $E_{e} \approx 32 \mathrm{MeV}$ for $\omega_{\mathrm{XFEL}}=15 \mathrm{keV}$. Even below that value, pair production is enabled by bandwidth effects, see Appendix A. If (by a yet unknown technology) the XFEL beam could be focused to achieve $\xi_{\text {XFEL }} \gtrsim 1$, a new domain of testing strong-field QED effects would be opened up, see the magenta region in Fig. 7 .

\section{Appendix A: Bandwidth effects in linear trident}

Turning to boost invariant quantities we describe the linearly polarized laser pulse by the electromagnetic four-potential $A=\left(A^{0}, \vec{A}\right)$ in axial gauge, $A^{0}=0$, where $\vec{A}=f(\phi) \vec{a}_{x} \cos \phi$ and the envelope function reads $f(\phi)=\cos ^{2}\left(\frac{\pi \phi}{2 \Delta \phi}\right) \sqcap(\phi, 2 \Delta \phi)$, i.e. the number of laser- field oscillations within the pulse is $N=\Delta \phi / \pi$. The invariant laser phase is $\phi=k \cdot x$. Due to the finite pulse duration bandwidth effects occur, since the power spectrum of the laser has frequency components $\lessgtr \omega$, thus enabling the sub-threshold pair production in the process XFEL $+e^{-} \rightarrow e^{-1}+e^{+} e^{-}$. The here assumed laser intensity parameter $\xi=e^{2} \vec{a}^{2} / m^{2} \ll 1$ is too small to allow for genuine multi-photon effects.

In Fig. 8 the inclusive differential positron cross section $d^{3} \sigma / d y d p_{\perp} d \varphi$ is exhibited, where $y, p_{\perp}$ and $\varphi$ denote the rapidity, the transverse momentum and azimuthal angle (w.r.t. to the polarization direction given by $\vec{a}_{x}$ ) of the produced positrons. The presented results are based on the weak-field evaluation of the l.h.s. Furry picture diagrams displayed in the introduction. We have selected three energies described by the Mandelstam variable $s=$ $(p+k)^{2}$. The threshold is given by $\sqrt{s}=3 m$. The left and middle panels are above and near threshold, while the right panel is below the threshold. The shorter the pulse, i.e. the smaller $\Delta \phi$, the wider becomes the kinematically accessible region. In the limit of long pulses, $\Delta \phi \gg 1$, the sub-threshold production is strongly suppressed.

The figure applies to any position on the respective diagonal lines in Fig. 7 for given value of $\sqrt{s}$ by shifting the rapidity $y \rightarrow y+y_{x}$ with $y_{x}=\ln \sqrt{s} / m$. The positron energy in the lab. frame, where $E_{e}=m \cosh y_{e}$, is $E^{+}=$ $\sqrt{m^{2}+p_{\perp}^{2}} \cosh \left(y+y_{x}+y_{e}\right)$.

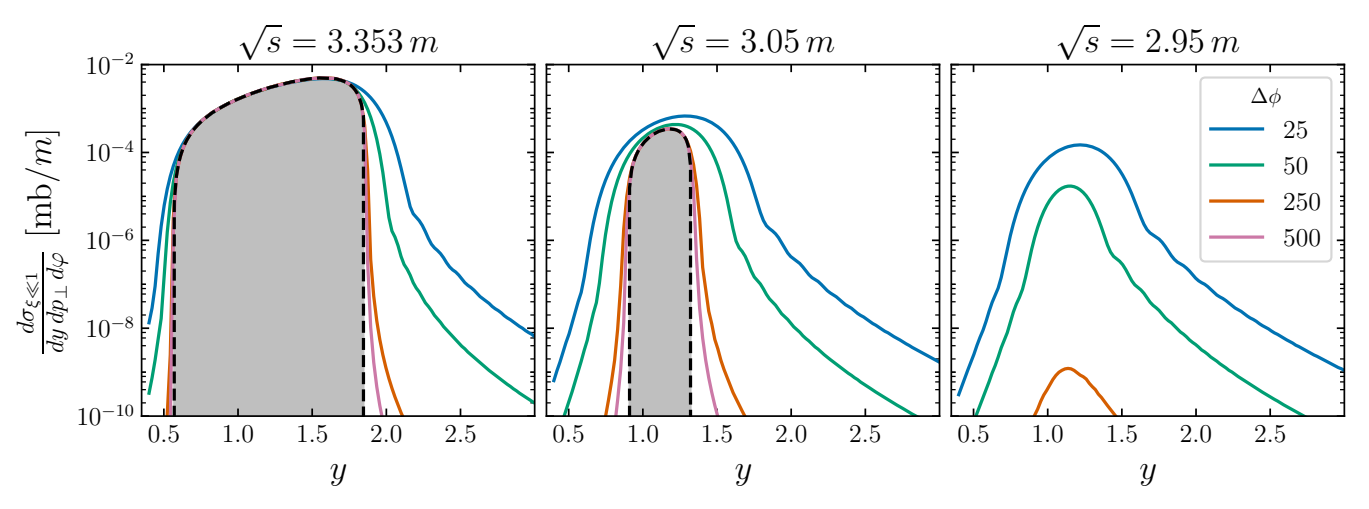

FIG. 8: (Color online) Inclusive differential positron cross section $d^{3} \sigma / d y d p_{\perp} d \varphi$ for $\sqrt{s} / m=3.353$ (left panel), 3.05 (middle panel) and 2.95 (right panel) as a function of rapidity $y$ for $p_{\perp} / m=0.15$ and $\varphi=0$. The pulse duration parameter is $\Delta \phi=25$ (blue), 50 (green), 250 (red) and 500 (magenta). The black dashed curves limiting the gray region in left and middle panels depict the case of a monochromatic beam, i.e. $\Delta \phi \rightarrow \infty$. Details of the employed formalism and the kinematic relations can be found in [45, 46].

\section{Appendix B: Testing EQ.(22) BY E-144 DATA}

The choice $F_{t}=T_{0}^{2} / 2$ with $T_{0}=2 \pi / \omega$ in Eq. 22 copes well with the $\xi$ dependence of E-144 data (see Fig. 99. Also the differential $E^{+}$dependence is sensi- tive to variations of $\xi$ (see Fig. 10). An effective value of $\xi=0.25$ is consistent with the positron momentum distribution of E-144 (see Fig. 11). 


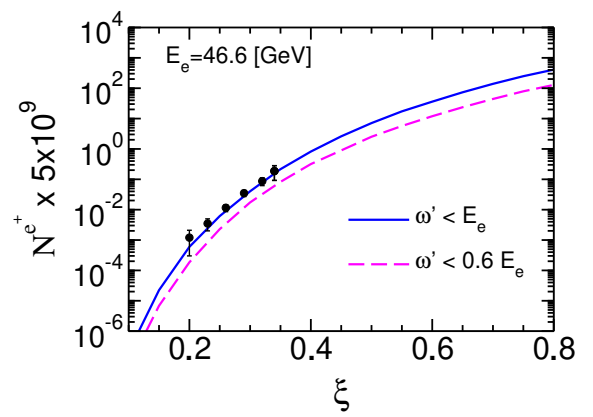

FIG. 9: (Color online) Positron number as a function of laser intensity parameter $\xi$ for $F_{t}=T_{0}^{2} / 2$ with $T_{0}=2 \pi / \omega$ and $5 \times 10^{9}$ electrons per bunch in Eq. 22, The data are from 25] (table 13). Accordingly the here employed laser frequency is $\omega=2.3527 \mathrm{eV}$.

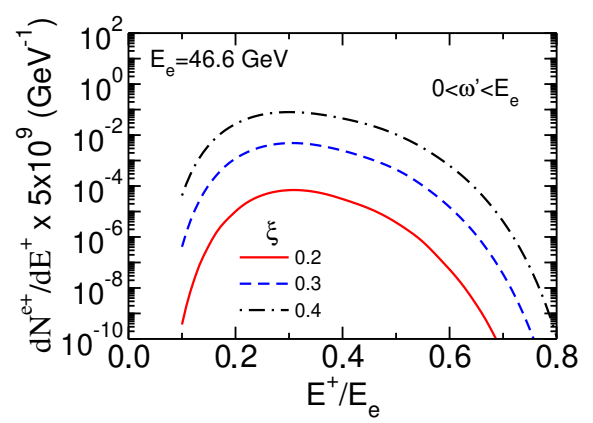

FIG. 10: (Color online) Differential positron spectrum $d N^{e^{+}} / d E^{+}$as a function of $E^{+} / E_{e}$ with the same normalization as in Fig. 9 for $\xi=0.2$ (red), 0.3 (blue dashed) and 0.4 (black dot-dashed). Despite the employed head-on geometry a value of $\xi=0.246$ reproduces the peak height of [13] (see figure 2 there) when imposing the multiplicative factor of $2 \times 10^{4}$ for the number of laser shots; our distribution is up shifted by about $2 \mathrm{GeV}$ and has a somewhat steeper l.h.s. flank.

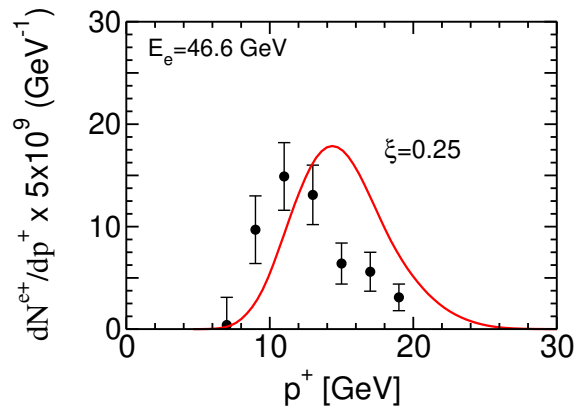

FIG. 11: (Color online) Differential positron spectrum $d N^{e^{+}} / d p^{+}$(red curve) as a function of the lab. positron momentum $p^{+}$with the same normalization as in Figs. 9 and 10 for $\xi=0.25$ (see also [14) and for 21,962 laser shots. Data from [25] (table 12). Including an inclination of $17^{\circ}$ between electron beam and laser beam shifts the red curve to left.

\section{Acknowledgments}

The authors gratefully acknowledge communications with G. Torgrimsson, A. DiPiazza and the longstanding collaboration with D. Seipt. One author (BK) thanks J. Z. Kaminski for explanations of the stabilization phenomenon. The work is supported by R. Sauerbrey and T. E. Cowan w.r.t. the study of fundamental QED processes for HIBEF. This work was partly funded by the Center for Advanced Systems Understanding (CASUS) that is financed by Germany's Federal Ministry of Education and Research (BMBF) and by the Saxon Ministry for Science, Culture and Tourism (SMWK) with tax funds on the basis of the budget approved by the Saxon State Parliament.
[1] E. Loetstedt and U. D. Jentschura, "Correlated twophoton emission by transitions of Dirac-Volkov states in intense laser fields: QED predictions," Phys. Rev. A 80, 053419 (2009) arXiv:0911.4765 [quant-ph]].

[2] D. Seipt and B. Kämpfer, "Two-photon Compton process in pulsed intense laser fields," Phys. Rev. D 85, 101701 (2012) arXiv:1201.4045 [hep-ph]].

[3] F. Mackenroth and A. Di Piazza, "Nonlinear Double Compton Scattering in the Ultrarelativistic Quantum Regime," Phys. Rev. Lett. 110, no.7, 070402 (2013) arXiv:1208.3424 [hep-ph]].

[4] A. Ilderton, "Trident pair production in strong laser pulses," Phys. Rev. Lett. 106, 020404 (2011) arXiv:1011.4072 [hep-ph]].

[5] B. King and H. Ruhl, "Trident pair production in a constant crossed field," Phys. Rev. D 88, no.1, 013005 (2013) arXiv:1303.1356 [hep-ph]].

[6] B. King, N. Elkina and H. Ruhl, "Photon polarisation in electron-seeded pair-creation cascades," Phys. Rev. A
87, 042117 (2013) arXiv:1301.7001 [hep-ph]].

[7] G. Torgrimsson, "Nonlinear trident in the highenergy limit: Nonlocality, Coulomb field and resummations," Phys. Rev. D 102, no. 9, 096008 (2020) arXiv:2007.08492 [hep-ph]].

[8] V. Dinu and G. Torgrimsson, "Approximating higherorder nonlinear QED processes with first-order building blocks," Phys. Rev. D 102, no.1, 016018 (2020) arXiv:1912.11015 [hep-ph]].

[9] V. Dinu and G. Torgrimsson, "Trident process in laser pulses," Phys. Rev. D 101, no. 5, 056017 (2020) arXiv:1912.11017 [hep-ph]].

[10] G. Torgrimsson, "Loops and polarization in strongfield QED," New J. Phys. 23, no.6, 065001 (2021) arXiv:2012.12701 [hep-ph]].

[11] V. Dinu and G. Torgrimsson, "Trident pair production in plane waves: Coherence, exchange, and spacetime inhomogeneity," Phys. Rev. D 97, no.3, 036021 (2018) arXiv:1711.04344 [hep-ph]]. 
[12] B. King and A. M. Fedotov, "Effect of interference on the trident process in a constant crossed field," Phys. Rev. D 98, no. 1, 016005 (2018) arXiv:1801.07300 [hep-ph]].

[13] F. Mackenroth and A. Di Piazza, "Nonlinear trident pair production in an arbitrary plane wave: a focus on the properties of the transition amplitude," Phys. Rev. D 98, no. 11, 116002 (2018) arXiv:1805.01731 [hep-ph]].

[14] H. Hu, C. Müller and C. H. Keitel, "Complete QED theory of multiphoton trident pair production in strong laser fields," Phys. Rev. Lett. 105, 080401 (2010) arXiv:1002.2596 [physics.atom-ph]].

[15] V. I. Ritus, "Vacuum polarization correction to elastic electron and muon scattering in an intense field and pair electro- and muoproduction," Nucl. Phys. B 44, 236 (1972)

[16] V. N. Baier, V. M. Katkov, V. M. Strakhovenko, "Higherorder effects in external fields: pair production by a particle," Sov. J. Nucl. Phys. 14, 572 (1972)

[17] A. Gonoskov et al., "Extended particle-in-cell schemes for physics in ultrastrong laser fields: Review and developments," Phys. Rev. E 92, no. 2, 023305 (2015) arXiv:1412.6426 [physics.plasm-ph]].

[18] A. Di Piazza, M. Tamburini, S. Meuren and C. H. Keitel, "Improved local-constant-field approximation for strongfield QED codes," Phys. Rev. A 99, no. 2, 022125 (2019) arXiv:1811.05834 [hep-ph]].

[19] A. Gonoskov, T. G. Blackburn, M. Marklund and S. S. Bulanov, "Charged particle motion and radiation in strong electromagnetic fields," arXiv:2107.02161 [physics.plasm-ph]].

[20] S. Meuren, "Probing Strong-field QED at FACET-II (SLAC E-320) (2019)",

https://conf.slac.stanford.edu/facet-2-2019/sites/ facet-2-2019. conf.slac.stanford.edu/files/ basic-page-docs/sfqed_2019.pdf .

[21] S. Meuren et al., "On Seminal HEDP Research Opportunities Enabled by Colocating Multi-Petawatt Laser with High-Density Electron Beams," arXiv:2002.10051 [physics.plasm-ph].

[22] H. Abramowicz et al., "Letter of Intent for the LUXE Experiment," arXiv:1909.00860 [physics.ins-det].

[23] H. Abramowicz et al., "Conceptual Design Report for the LUXE Experiment," arXiv:2102.02032 [hep-ex].

[24] F. C. Salgado, N. Cavanagh, M. Tamburini, D. W. Storey, R. Beyer, P. H. Bucksbaum, Z. Chen, A. Di Piazza, E. Gerstmayr and Harsh, et al. "Single Particle Detection System for Strong-Field QED Experiments," arXiv:2107.03697 [hep-ex]].

[25] C. Bamber et al., "Studies of nonlinear QED in collisions of 46.6-GeV electrons with intense laser pulses," Phys. Rev. D 60, 092004 (1999).

[26] D. L. Burke et al., "Positron production in multi - photon light by light scattering," Phys. Rev. Lett. 79, 1626 (1997).

[27] J. M. Cole et al., "Experimental evidence of radiation reaction in the collision of a high-intensity laser pulse with a laser-wakefield accelerated electron beam," Phys. Rev. X 8, no. 1, 011020 (2018) arXiv:1707.06821 [physics.plasm$\mathrm{ph}]$.

[28] K. Poder et al., "Experimental Signatures of the Quantum Nature of Radiation Reaction in the Field of an Ultraintense Laser," Phys. Rev. X 8, no. 3, 031004 (2018) arXiv:1709.01861 [physics.plasm-ph]].
[29] N. V. Elkina et al., "QED cascades induced by circularly polarized laser fields," Phys. Rev. ST Accel. Beams 14, 054401 (2011), arXiv:1010.4528 [hep-ph]].

[30] D. Seipt, C. P. Ridgers, D. Del Sorbo and A. G. R. Thomas, "Polarized QED cascades," New J. Phys. 23, no.5, 053025 (2021) arXiv:2010.04078 [hep-ph]].

[31] B. King, "Uniform locally constant field approximation for photon-seeded pair production," Phys. Rev. A 101, no.4, 042508 (2020) arXiv:1908.06985 [hep-ph]].

[32] B. King, "Interference effects in nonlinear Compton scattering due to pulse envelope," Phys. Rev. D 103, no.3, 036018 (2021) arXiv:2012.05920 [hep-ph]].

[33] U. Hernandez Acosta, A. I. Titov and B. Kämpfer, "Rise and fall of laser-intensity effects in spectrally resolved Compton process," New. J. Phys. 23, 095008 (2021)/

[34] V. I. Ritus, "Quantum effects of the interaction of elementary particles with an intense electromagnetic field". J. Sov. Laser Res. (United States), 6:5, 497 (1985).

[35] U. Hernandez Acosta, A. Otto, B. Kämpfer and A. I. Titov, "Nonperturbative signatures of nonlinear Compton scattering," Phys. Rev. D 102, no.11, 116016 (2020) arXiv:2001.03986 [hep-ph]].

[36] B. Kämpfer and A. I. Titov, "Impact of laser polarization on q-exponential photon tails in non-linear Compton scattering," Phys. Rev. A 103, 033101 (2021) arXiv:2012.07699 [hep-ph]].

[37] S. Tang and B. King, "Pulse envelope effects in nonlinear Breit-Wheeler pair-creation," Phys. Rev. D 104096019 (2021).

[38] As alternative to the above approach which deploys rates, one could use the convolution in (1) without any time ordering by turning to probabilities per pulse, analog to the "product approach" in [5].

[39] J. Z. Kamiński, K. Krajewska and F. Ehlotzky, "Monte Carlo analysis of electron-positron pair creation by powerful laser-ion impact," Phys. Rev. A 74, no.3, 033402 (2006)

[40] A. Hartin, A. Ringwald and N. Tapia, "Measuring the Boiling Point of the Vacuum of Quantum Electrodynamics," Phys. Rev. D 99, no.3, 036008 (2019) arXiv:1807.10670 [hep-ph]].

[41] H. R. Reiss, "Production of electron pairs from a zeromass state," Phys. Rev. Lett. 26, 1072-1075 (1971)

[42] K. Fleck, N. Cavanagh and G. Sarri, "Conceptual Design of a High-flux Multi-GeV Gamma-ray Spectrometer," Sci. Rep. 10, no. 1, 9894 (2020).

[43] HIBEF: Helmholtz International Beam Line for Extreme Fields, cf. https: //www.hzdr.de/db/Cms?p0id=50566\&pNid=694

[44] D. Seipt and B. Kämpfer, "Laser assisted Compton scattering of X-ray photons," Phys. Rev. A 89, no.2, 023433 (2014) arXiv:1309.2092 [physics.atom-ph]].

[45] U. Hernandez Acosta and B. Kämpfer, "Laser pulselength effects in trident pair production," Plasma Phys. Control. Fusion 61, no.8, 084011 (2019) arXiv:1901.08860 [hep-ph]].

[46] U. Hernandez Acosta, "Pulsed perturbative QED: a study of trident pair production in pulsed laser fields," PhD thesis, TU Dresden, Germany (2020); https://nbn-resolving.org/urn:nbn:de: bsz:14-qucosa2-760352. 